\title{
Fixed points and endpoints of contractive set-valued maps in cone uniform spaces with generalized pseudodistances
}

\author{
Kazimierz Włodarczyk ${ }^{*}$ and Robert Plebaniak
}

\section{"Correspondence:}

wlkzxa@math.uni.lodz.pl

Department of Nonlinear Analysis,

Faculty of Mathematics and

Computer Science, University of

Łódź, Banacha 22, Łódź, 90-238,

Poland

\begin{abstract}
We introduce the concept of contractive set-valued maps in cone uniform spaces with generalized pseudodistances and we show how in these spaces our fixed point and endpoint existence theorem of Caristi type yields the fixed point and endpoint existence theorem for these contractive maps.

MSC: 47H10; 54C60; 47H09; 54E15; 46A03; 54E50; 46B40

Keywords: fixed point; endpoint; contraction of Nadler type; cone uniform space; generalized pseudodistance; set-valued dynamic system; dynamic process
\end{abstract}

\section{Introduction}

Nadler [1] extended Banach's fixed point theorem [2] for set-valued maps in complete metric spaces.

Theorem 1.1 ([1, Th. 5$])$ Let $(X, d)$ be a complete metric space, let $\mathrm{Cl}(X)$ denote the class of all nonempty closed subsets of $X$, and let $H:(\mathrm{Cl}(X))^{2} \rightarrow[0, \infty]$ be defined by

$$
\forall_{A, B \in \mathrm{Cl}(X)}\left\{H(A, B)=\max \left\{\sup _{u \in A} d(u, B), \sup _{v \in B} d(v, A)\right\}\right.
$$

where, for each $u \in X$ and $V \in \mathrm{Cl}(X), d(u, V)=\inf _{v \in V} d(u, v)$. If a set-valued map $T: X \rightarrow$ $\mathrm{Cl}(X)$ is $H$-contractive, i.e., if $T$ satisfies

$$
\exists_{0<\lambda<1} \forall_{x, y \in X}\{H(T(x), T(y)) \leq \lambda d(x, y)\}
$$

then $T$ has a fixed point $w$ in $X$, i.e., $w \in T(w)$.

A number of authors introduce the new concepts of set-valued contractions of Nadler type and study the problem concerning the existence of fixed points for such contractions; see, e.g., Aubin and Siegel [3], de Blasi et al. [4], Ćirić [5], Eldred et al. [6], Feng and Liu [7], Frigon [8], Al-Homidan et al. [9], Jachymski [10], Kaneko [11], Klim and Wardowski [12], Latif and Al-Mezel [13], Mizoguchi and Takahashi [14], Pathak and Shahzad [15], Quantina and Kamran [16], Reich [17, 18], Reich and Zaslavski [19, 20], Sintunavarat and Kumam [21-25], Suzuki [26], Suzuki and Takahashi [27], Takahashi [28] and Zhong et al. [29]. In particular, the significant fixed point existence results of Nadler type were obtained 
by Suzuki [30, Th. 3.7] in metric spaces with $\tau$-distances and by Wardowski [31] in cone metric spaces.

Recently, Włodarczyk and Plebaniak in [32] have studied among others the $\mathcal{J}$-families of generalized pseudodistances in cone uniform, uniform and metric spaces which generalize distances of Tataru [33], $w$-distances of Kada et al. [34], $\tau$-distances of Suzuki [35] and $\tau$-functions of Lin and Du [36] in metric spaces and distances of Vályi [37] in uniform spaces.

In the present paper, we introduce the concept of contractive set-valued maps in cone uniform spaces with generalized pseudodistances, and we show how in these spaces our fixed point and endpoint existence theorem of Caristi type [32, Th. 4.5] yields the fixed point and endpoint existence theorem for these contractive maps.

It is worth noticing that our fixed point and endpoint existence Theorem 3.1: has a simpler proof; is Nadler type; is new in cone uniform and cone locally convex spaces; is new even in cone metric and metric spaces; and is different from those given in the previous publications on this subject.

This paper is a continuation of [32, 38-46].

\section{Definitions and notations}

We define a real normed space to be a pair $(L,\|\cdot\|)$ with the understanding that a vector space $L$ over $\mathbb{R}$ carries the topology generated by the metric $(a, b) \rightarrow\|a-b\|, a, b \in L$.

A nonempty closed convex set $H \subset L$ is called a cone in $L$ if it satisfies:

(H1) $\forall_{s \in(0, \infty)}\{s H \subset H\}$;

(H2) $H \cap(-H)=\{0\}$; and

(H3) $H \neq\{0\}$.

It is clear that each cone $H \subset L$ defines, by virtue of

$$
\text { " } a \preceq_{H} b \text { iff } b-a \in H \text { ", }
$$

an order of $L$ under which $L$ is an ordered normed space with a cone $H$.

We will write $a \prec_{H} b$ to indicate that $a \preceq_{H} b$, but $a \neq b$. A cone $H$ is said to be solid if $\operatorname{int}(H) \neq \varnothing ; \operatorname{int}(H)$ denotes the interior of $H$. We will write $a \ll_{H} b$ to indicate that $b-a \in$ $\operatorname{int}(H)$.

The cone $H$ is normal if a real number $M>0$ such that for each $a, b \in H, 0 \preceq_{H} a \preceq_{H} b$ implies $\|a\| \leq M\|b\|$ exists. The number $M$ satisfying above is called the normal constant of $H$.

Let an element $+\infty \notin L$ be such that $a \preceq_{H}+\infty$ for all $a \in L$.

Let $2^{X}$ denote the family of all nonempty subsets of a space $X$. Recall that a set-valued dynamic system is defined as a pair $(X, T)$, where $X$ is a certain space and $T$ is a set-valued map $T: X \rightarrow 2^{X}$; in particular, a set-valued dynamic system includes the usual dynamic system where $T$ is a single-valued map. We say that a map $\omega: X \rightarrow L \cup\{+\infty\}$ is proper if its effective domain, $\operatorname{dom}(\omega)=\{x: \omega(x) \neq+\infty\}$, is nonempty.

Definition 2.1 ([38, Def. 2.2]) Let $X$ be a nonempty set, and let $L$ be an ordered normed space with a cone $H$.

(i) The family $\mathcal{P}=\left\{p_{\alpha}: X^{2} \rightarrow L, \alpha \in \mathcal{A}\right\}, \mathcal{A}$-index set, is said to be a $\mathcal{P}$-family of cone pseudometrics on $X$ ( $\mathcal{P}$-family for short) if the following three conditions hold: 
$(\mathcal{P} 1) \forall_{\alpha \in \mathcal{A}} \forall_{x, y \in X}\left\{0 \preceq_{H} p_{\alpha}(x, y) \wedge x=y \Rightarrow p_{\alpha}(x, y)=0\right\}$;

$(\mathcal{P} 2) \forall_{\alpha \in \mathcal{A}} \forall_{x, y \in X}\left\{p_{\alpha}(x, y)=p_{\alpha}(y, x)\right\}$; and

(P3) $\forall_{\alpha \in \mathcal{A}} \forall_{x, y, z \in X}\left\{p_{\alpha}(x, z) \preceq_{H} p_{\alpha}(x, y)+p_{\alpha}(y, z)\right\}$.

(ii) If $\mathcal{P}$ is a $\mathcal{P}$-family, then the pair $(X, \mathcal{P})$ is called a cone uniform space.

(iii) A $\mathcal{P}$-family $\mathcal{P}$ is said to be separating if

$(\mathcal{P} 4) \forall_{x, y \in X}\left\{x \neq y \Rightarrow \exists_{\alpha \in \mathcal{A}}\left\{0 \prec_{H} p_{\alpha}(x, y)\right\}\right\}$.

(iv) If a $\mathcal{P}$-family $\mathcal{P}$ is separating, then the pair $(X, \mathcal{P})$ is called a Hausdorff cone uniform space.

Definition 2.2 ([38, Def. 2.3]) Let $L$ be an ordered normed space with a solid cone $H$, and let $(X, \mathcal{P})$ be a Hausdorff cone uniform space with a cone $H$.

(i) We say that a sequence $\left(w_{m}: m \in \mathbb{N}\right)$ in $X$ is a $\mathcal{P}$-convergent in $X$ (convergent in $X$ for short) if there exists $w \in X$ such that

$$
\forall_{\alpha \in \mathcal{A}} \forall_{c_{\alpha} \in L, 0 \ll c_{\alpha}} \exists_{n_{0}=n_{0}\left(\alpha, c_{\alpha}\right) \in \mathbb{N}} \forall_{m \in \mathbb{N} ; n_{0} \leq m}\left\{p_{\alpha}\left(w_{m}, w\right) \ll_{H} c_{\alpha}\right\} .
$$

(ii) We say that a sequence $\left(w_{m}: m \in \mathbb{N}\right)$ in $X$ is a $\mathcal{P}$-Cauchy sequence in $X$ (Cauchy sequence in $X$, for short) if

$$
\forall_{\alpha \in \mathcal{A}} \forall_{c_{\alpha} \in L, 0 \ll c_{\alpha}} \exists_{n_{0}=n_{0}\left(\alpha, c_{\alpha}\right) \in \mathbb{N}} \forall_{m, n \in \mathbb{N} ; n_{0} \leq m<n}\left\{p_{\alpha}\left(w_{m}, w_{n}\right) \ll_{H} c_{\alpha}\right\}
$$

(iii) If every Cauchy sequence in $X$ is convergent in $X$, then $(X, \mathcal{P})$ is called a $\mathcal{P}$-sequentially complete cone uniform space (sequentially complete for short).

Theorem 2.1 ([32, Th. 2.3]) Let L be an ordered Banach space with a normal solid cone $H$, and let $(X, \mathcal{P})$ be a Hausdorff cone uniform space with a cone $H$. The following hold:

(P1) The sequence $\left(w_{m}: m \in \mathbb{N}\right)$ in $X$ converges to $w \in X$ iff

$$
\forall_{\alpha \in \mathcal{A}} \forall_{\varepsilon_{\alpha}>0} \exists_{n_{0} \in \mathbb{N}} \forall_{m \in \mathbb{N} ; m \geq n_{0}}\left\{\left\|p_{\alpha}\left(w_{m}, w\right)\right\|<\varepsilon_{\alpha}\right\}
$$

(P2) The sequence $\left(w_{m}: m \in \mathbb{N}\right)$ in $X$ is a Cauchy sequence in $X$ iff

$$
\forall_{\alpha \in \mathcal{A}} \forall_{\varepsilon_{\alpha}>0} \exists_{n_{0} \in \mathbb{N}} \forall_{m, n \in \mathbb{N} ; m>n \geq n_{0}}\left\{\left\|p_{\alpha}\left(w_{m}, w_{n}\right)\right\|<\varepsilon_{\alpha}\right\}
$$

Definition 2.3 Let $L$ be an ordered Banach space with a cone $H$.

(i) A subset $D \subset L$ is said to have a minimal (maximal) element if there exists $a \in D$ such that $a \preceq_{H} b\left(b \preceq_{H} a\right)$ for all $b \in D$, and we write then that $a=\min (D)$ $(a=\max (D))$. It is clear that if $D$ has a minimal (maximal) element, then the minimal (maximal) element is unique.

(ii) We say that $a \in L$ is an infimum (supremum) for set $D \subset L$ if $\operatorname{cl}_{L}(D)$ has the minimal (maximal) element and $a=\min \left(\operatorname{cl}_{L}(D)\right)\left(a=\max \left(\operatorname{cl}_{L}(D)\right)\right)$, and we write then that $a=\inf (D)(a=\sup (D))$; here $\operatorname{cl}_{L}(D)$ denotes the closure of $D$ in $L$.

Definition 2.4 Let $L$ be an ordered normed space with a solid cone $H$. The cone $H$ is called regular if for every increasing (decreasing) sequence $\left(c_{m}: m \in \mathbb{N}\right)$ in $L$ which is 
bounded from above (below),

$$
\begin{aligned}
& \left(\text { i.e. }, c_{1} \preceq_{H} c_{2} \preceq_{H} \cdots \preceq_{H} c_{m} \preceq_{H} \cdots \preceq_{H} b\right. \\
& \left.\left(b \preceq_{H} \cdots \preceq_{H} c_{m} \preceq_{H} \cdots \preceq_{H} c_{2} \preceq_{H} c_{1}\right) \text { for some } b \in L\right),
\end{aligned}
$$

there exists $c \in L$ such that $\lim _{m \rightarrow \infty}\left\|c_{m}-c\right\|=0$. Every regular cone is normal.

Definition 2.5 ([32, Def. 2.6]) Let $L$ be an ordered normed space with a normal solid cone $H$, and let $(X, \mathcal{P})$ be a Hausdorff cone uniform space with a cone $H$.

(i) The family $\mathcal{J}=\left\{J_{\alpha}: X^{2} \rightarrow L, \alpha \in \mathcal{A}\right\}$ is said to be a $\mathcal{J}$-family of cone pseudodistances on $X$ (J-family on $X$ for short) if the following three conditions hold:

$(\mathcal{J} 1) \forall_{\alpha \in \mathcal{A}} \forall_{x, y \in X}\left\{0 \preceq_{H} J_{\alpha}(x, y)\right\}$;

(J2) $\forall_{\alpha \in \mathcal{A}} \forall_{x, y, z \in X}\left\{J_{\alpha}(x, z) \preceq_{H} J_{\alpha}(x, y)+J_{\alpha}(y, z)\right\}$; and

$(\mathcal{J} 3)$ For any sequence $\left(w_{m}: m \in \mathbb{N}\right)$ in $X$ such that

$$
\forall_{\alpha \in \mathcal{A}} \forall_{\varepsilon_{\alpha}>0} \exists_{n_{0}=n_{0}\left(\alpha, \varepsilon_{\alpha}\right) \in \mathbb{N}} \forall_{m, n \in \mathbb{N} ; n_{0} \leq m \leq n}\left\{\left\|J_{\alpha}\left(w_{m}, w_{n}\right)\right\|<\varepsilon_{\alpha}\right\},
$$

if there exists a sequence $\left(v_{m}: m \in \mathbb{N}\right)$ in $X$ satisfying

$$
\forall_{\alpha \in \mathcal{A}} \forall_{\varepsilon_{\alpha}>0} \exists_{n_{0}=n_{0}\left(\alpha, \varepsilon_{\alpha}\right) \in \mathbb{N}} \forall_{m \in \mathbb{N} ; n_{0} \leq m}\left\{\left\|J_{\alpha}\left(w_{m}, v_{m}\right)\right\|<\varepsilon_{\alpha}\right\},
$$

then

$$
\forall_{\alpha \in \mathcal{A}} \forall_{\varepsilon_{\alpha}>0} \exists_{n_{0}=n_{0}\left(\alpha, \varepsilon_{\alpha}\right) \in \mathbb{N}} \forall_{m \in \mathbb{N} ; n_{0} \leq m}\left\{\left\|p_{\alpha}\left(w_{m}, v_{m}\right)\right\|<\varepsilon_{\alpha}\right\} .
$$

(ii) Each $\mathcal{P}$-family is a $\mathcal{J}$-family.

(iii) If $\mathcal{J}=\left\{J_{\alpha}: X^{2} \rightarrow L: \alpha \in \mathcal{A}\right\}$ is a $\mathcal{J}$-family, then $X=X_{\mathcal{J}}^{0} \cup X_{\mathcal{J}}^{+}$where

$$
X_{\mathcal{J}}^{0}=\left\{x \in X: \forall_{\alpha \in \mathcal{A}}\left\{0=J_{\alpha}(x, x)\right\}\right\}
$$

and

$$
X_{\mathcal{J}}^{+}=\left\{x \in X: \exists_{\alpha \in \mathcal{A}}\left\{0 \prec_{H} J_{\alpha}(x, x)\right\}\right\}
$$

Let $(X, \mathcal{P})$ be a sequentially complete cone uniform space. We say that a set $Y \in 2^{X}$ is closed in $X$ if $Y=\operatorname{cl}_{X}(Y)$ where $\operatorname{cl}_{X}(Y)$, the closure of $Y$ in $X$, denotes the set of all $w \in X$ for which there exists a sequence $\left(w_{m}: m \in \mathbb{N}\right)$ in $Y$ which converges to $w$. If a set $Y \in 2^{X}$ is closed in $X$, then $(Y, \mathcal{P})$ is a sequentially complete cone uniform space with a cone $H$. Define $\mathrm{Cl}(X)=\left\{Y \in 2^{X}: Y=\mathrm{cl}_{X}(Y)\right\}$; that is, $\mathrm{Cl}(X)$ denotes the class of all nonempty closed subsets of $X$.

Definition 2.6 Let $L$ be an ordered Banach space with a normal solid cone $H$, let $(X, \mathcal{P})$ be a Hausdorff sequentially complete cone uniform space with a cone $H$, and let $\mathcal{J}=\left\{J_{\alpha}\right.$ : $X^{2} \rightarrow L, \alpha \in \mathcal{A}$ \} be a $\mathcal{J}$-family.

(i) Let $A, B \in \mathrm{Cl}(X)$. We say that a pair $(A, B)$ is $\mathcal{J}$-admissible if: 
(a) For each $\alpha \in \mathcal{A}, x \in A$ and $y \in B$, the $\operatorname{set} \operatorname{cl}_{L}\left(\left\{J_{\alpha}(x, v): v \in B\right\}\right)$ has a minimal element, say $J_{\alpha}(x, B)$ (i.e., $J_{\alpha}(x, B)=\inf _{v \in B} J_{\alpha}(x, v)$ ), and the set $\operatorname{cl}_{L}\left(\left\{J_{\alpha}(y, u): u \in A\right\}\right)$ has a minimal element, say $J_{\alpha}(y, A)($ i.e., $\left.J_{\alpha}(y, A)=\inf _{u \in A} J_{\alpha}(y, u)\right)$;

(b) The sets $\mathrm{cl}_{L}\left(\left\{J_{\alpha}(u, B): u \in A\right\}\right)$ and $\mathrm{cl}_{L}\left(\left\{J_{\alpha}(v, A): v \in B\right\}\right)$ have maximal elements, say $J_{\alpha}(A, B)$ and $J_{\alpha}(B, A)$, respectively (i.e.,

$$
J_{\alpha}(A, B)=\sup _{u \in A} J_{\alpha}(u, B)=\sup _{u \in A} \inf _{v \in B} J_{\alpha}(u, v)
$$

and

$$
J_{\alpha}(B, A)=\sup _{v \in B} J_{\alpha}(v, A)=\sup _{v \in B} \inf _{u \in A} J_{\alpha}(v, u)
$$

respectively); and

(c) For each $\alpha \in \mathcal{A}$, the elements $J_{\alpha}(A, B)$ and $J_{\alpha}(B, A)$ are comparable.

(ii) Let $A, B \in \mathrm{Cl}(X)$, and let a pair $(A, B)$ be $\mathcal{J}$-admissible. For each $\alpha \in \mathcal{A}$, we define $\mathcal{H}^{\mathcal{J}}=\left\{H_{\alpha}^{\mathcal{J}}(A, B), \alpha \in \mathcal{A}\right\}$ where

$$
\forall_{\alpha \in \mathcal{A}}\left\{H_{\alpha}^{\mathcal{J}}(A, B)=\max \left\{J_{\alpha}(A, B), J_{\alpha}(B, A)\right\}\right\}
$$

Here, for each $\alpha \in \mathcal{A}, H_{\alpha}^{\mathcal{J}}(A, B) \in L \cup\{+\infty\}$ and by $(\mathcal{J} 1)$ and since $H$ is closed, $0 \preceq_{H} H_{\alpha}^{\mathcal{J}}(A, B)$.

(iii) Let a set-valued dynamic system $(X, T)$ satisfy $T: X \rightarrow \operatorname{Cl}(X)$. We say that $(X, T)$ is $\mathcal{J}$-admissible if for each $x, y \in X$, a pair $(T(x), T(y))$ is $\mathcal{J}$-admissible.

(iv) Let $(X, T)$ satisfy $T: X \rightarrow \mathrm{Cl}(X)$, and let $(X, T)$ be $\mathcal{J}$-admissible. If there exists the family $\Lambda=\left\{\lambda_{\alpha} \in(0,1), \alpha \in \mathcal{A}\right\}$ such that

$$
\forall_{\alpha \in \mathcal{A}} \forall_{x, y \in X}\left\{H_{\alpha}^{\mathcal{J}}(T(x), T(y)) \preceq_{H} \lambda_{\alpha} J_{\alpha}(x, y)\right\}
$$

then we say that $(X, T)$ is $\mathcal{H}_{\Lambda}^{\mathcal{J}}$-contractive.

(v) Let $E \subseteq X, E \neq \varnothing$. The map $F: E \rightarrow H \cup\{+\infty\}$ is lower semicontinuous on $E$ with respect to $X$ (written: $F$ is $(E, X)$-lsc when $E \neq X$ and $F$ is $l s c$ when $E=X$ ) if the set $\left\{y \in E: F(y) \preceq_{H} c\right\}$ is a closed subset in $X$ for each $c \in H$. Equivalently, for each $x_{0} \in E$,

$$
F\left(x_{0}\right) \preceq_{H} \liminf _{x \rightarrow x_{0}, x \in X} F(x)
$$

(vi) We say that the family $\mathcal{J}$ is continuous in $X$ if for each $x_{0} \in X$ and for each sequence $\left(x_{m}: m \in \mathbb{N}\right)$ in $X$ converging to $x_{0}$, we have

$$
\forall_{\alpha \in \mathcal{A}}\left\{\lim _{m \rightarrow \infty} J_{\alpha}\left(x_{m}, x_{0}\right)=\lim _{m \rightarrow \infty} J_{\alpha}\left(x_{0}, x_{m}\right)=0\right\} .
$$

If $\mathcal{J}=\mathcal{P}$, then $\mathcal{J}$ is continuous in $X$. 


\section{Statement of result}

Let $(X, T)$ be a set-valued dynamic system. By $\operatorname{Fix}(T)$ and $\operatorname{End}(T)$ we denote the sets of all fixed points and endpoints of $T$, respectively, i.e., $\operatorname{Fix}(T)=\{w \in X: w \in T(w)\}$ and $\operatorname{End}(T)=$ $\{w \in X:\{w\}=T(w)\}$. A dynamic process or a trajectory starting at $w_{0} \in X$ or a motion of the system $(X, T)$ at $w_{0}$ is a sequence $\left(w_{m}: m \in\{0\} \cup \mathbb{N}\right)$ defined by $w_{m} \in T\left(w_{m-1}\right)$ for $m \in \mathbb{N}$ (see, Aubin-Siegel [3] and Yuan [47]).

The aim of this paper is to prove the following fixed point and endpoint existence general result of Nadler type.

Theorem 3.1 (i) Assume that:

(A1) $L$ is an ordered Banach space with a regular solid cone $H$;

(A2) $(X, \mathcal{P})$ is a Hausdorff sequentially complete cone uniform space with a cone $H$;

(A3) $\mathcal{J}=\left\{J_{\alpha}: X^{2} \rightarrow L, \alpha \in \mathcal{A}\right\}$ is a $\mathcal{J}$-family on $X$ such that $X_{\mathcal{J}}^{0} \neq \emptyset$;

(A4) The set-valued dynamic system $(X, T)$ satisfies $T: X \rightarrow \mathrm{Cl}(X)$ and is $\mathcal{J}$-admissible;

(A5) There exists the family $\Lambda=\left\{\lambda_{\alpha} \in(0,1), \alpha \in \mathcal{A}\right\}$ such that $(X, T)$ is $\mathcal{H}_{\Lambda}^{\mathcal{J}}$-contractive;

(A6) For each $x \in X$, the set $Q_{\mathcal{J} ; T}(x)$ is of the form:

$$
\begin{aligned}
Q_{\mathcal{J} ; T}(x)= & \left\{y \in T(x) \cap X_{\mathcal{J}}^{0}: \forall_{\alpha \in \mathcal{A}}\left\{J_{\alpha}(y, T(y))\right.\right. \\
& \left.\left.+\left(\gamma_{\alpha}-\lambda_{\alpha}\right) J_{\alpha}(x, y) \preceq_{H} J_{\alpha}(x, T(x))\right\}\right\},
\end{aligned}
$$

where the family $\Gamma=\left\{\gamma_{\alpha} \in(0,1), \alpha \in \mathcal{A}\right\}$ satisfies $\forall_{\alpha \in \mathcal{A}}\left\{\lambda_{\alpha}<\gamma_{\alpha}\right\}$;

(A7) For each $x \in X_{\mathcal{J}}^{0}$, the set $Q_{\mathcal{J} ; T}(x)$ is a nonempty subset in $X$; and

(A8) For each $x \in X_{\mathcal{J}}^{0}$, the set $Q_{\mathcal{J} ; T}(x)$ is a closed subset in $X$.

Then the following hold:

$\left(\mathrm{a}_{1}\right) \operatorname{Fix}(T) \neq \varnothing ;$ and

$\left(\mathrm{a}_{2}\right)$ For each $w \in \operatorname{Fix}(T), \forall_{\alpha \in \mathcal{A}}\left\{J_{\alpha}(w, w)=0\right\}$.

(ii) Assume, in addition, that:

(A9) For each $x \in X_{\mathcal{J}}^{0}$, each dynamic process $\left(w_{m}: m \in\{0\} \cup \mathbb{N}\right)$ starting at $w_{0}=x$ and satisfying $\forall_{m \in\{0\} \cup \mathbb{N}}\left\{w_{m+1} \in T\left(w_{m}\right)\right\}$ satisfies $\forall_{m \in\{0\} \cup \mathbb{N}}\left\{w_{m+1} \in Q_{\mathcal{J} ; T}\left(w_{m}\right)\right\}$.

Then the assertions $\left(\mathrm{a}_{1}\right)$ and $\left(\mathrm{a}_{2}\right)$ are of the forms:

$\left(\mathrm{a}_{1}^{\prime}\right) \operatorname{End}(T) \neq \varnothing$; and

$\left(\mathrm{a}_{2}^{\prime}\right)$ For each $w \in \operatorname{End}(T), \forall_{\alpha \in \mathcal{A}}\left\{J_{\alpha}(w, w)=0\right\}$.

Remark 3.1 (i) Assume that:

(A10) $\forall_{x \in X_{\mathcal{J}}^{0}}\left\{\left\{y \in T(x) \cap X_{\mathcal{J}}^{0}: \forall_{\alpha \in \mathcal{A}}\left\{\gamma_{\alpha} J_{\alpha}(x, y) \preceq_{H} J_{\alpha}(x, T(x))\right\}\right\} \neq \varnothing\right\}$.

Then (A7) holds.

(ii) Assume that one of the following conditions holds:

(A11) For each $(x, \alpha) \in X_{\mathcal{J}}^{0} \times \mathcal{A}$, the map

$$
J_{\alpha}(\cdot, T(\cdot))+\left(\gamma_{\alpha}-\lambda_{\alpha}\right) J_{\alpha}(x, \cdot): T(x) \cap X_{\mathcal{J}}^{0} \rightarrow H \cup\{+\infty\}
$$

is $\left(T(x) \cap X_{\mathcal{J}}^{0}, X\right)$-lsc;

(A12) The family $\mathcal{J}$ is continuous in $X$.

Then (A8) holds. 


\section{Proof of Theorem 3.1}

We will use the following fixed point and endpoint existence general result of Caristi type.

Theorem 4.1 ([32, Th. 4.5 ]) (i) Assume that:

(C1) $L$ is an ordered Banach space with a regular solid cone $H$;

$(\mathrm{C} 2)(X, \mathcal{P})$ is a Hausdorff sequentially complete cone uniform space with a cone $H$;

(C3) The family $\mathcal{J}=\left\{J_{\alpha}: X^{2} \rightarrow L, \alpha \in \mathcal{A}\right\}$ is a $\mathcal{J}$-family on $X$ such that $X_{\mathcal{J}}^{0} \neq \emptyset$;

(C4) The family $\Omega=\left\{\omega_{\alpha}: X \rightarrow H \cup\{+\infty\}, \alpha \in \mathcal{A}\right\}$ satisfies $D_{\Omega}=\bigcap_{\alpha \in \mathcal{A}} \operatorname{dom}\left(\omega_{\alpha}\right) \neq \varnothing$;

(C5) $(X, T)$ is a set-valued dynamic system;

(C6) $\left\{\varepsilon_{\alpha}, \alpha \in \mathcal{A}\right\}$ is a family of finite positive numbers;

(C7) For each $x \in X$, the set $Q_{\mathcal{J}, \Omega ; T}(x)$ is of the form:

$$
Q_{\mathcal{J}, \Omega ; T}(x)=\left\{y \in T(x) \cap X_{\mathcal{J}}^{0}: \forall_{\alpha \in \mathcal{A}}\left\{\omega_{\alpha}(y)+\varepsilon_{\alpha} J_{\alpha}(x, y) \preceq_{H} \omega_{\alpha}(x)\right\}\right\}
$$

(C8) For each $x \in X_{\mathcal{J}}^{0}$, the set $Q_{\mathcal{J}, \Omega ; T}(x)$ is a nonempty subset of $X$; and

(C9) For each $x \in X_{\mathcal{J}}^{0}$, the set $Q_{\mathcal{J}, \Omega ; T}(x)$ is a closed subset in $X$.

Then there exists $w \in D_{\Omega} \cap X_{\mathcal{J}}^{0}$ such that

(c) $w \in T(w)$.

(ii) Assume, in addition, that:

(C10) For each $x \in X_{\mathcal{J}}^{0}$, each dynamic process $\left(w_{m}: m \in\{0\} \cup \mathbb{N}\right)$ starting at $w_{0}=x$ and satisfying $\forall_{m \in\{0\} \cup \mathbb{N}}\left\{w_{m+1} \in T\left(w_{m}\right)\right\}$ satisfies $\forall_{m \in\{0\} \cup \mathbb{N}}\left\{w_{m+1} \in Q_{\mathcal{J}, \Omega ; T}\left(w_{m}\right)\right\}$.

Then assertion (c) is of the form:

$\left(\mathrm{c}^{\prime}\right)\{w\}=T(w)$

Remark 4.1 ([32, Remark 4.6 ]) (i) A special case of condition (C9) is a condition (C9') defined by:

$\left(\mathrm{C}^{\prime}\right)$ For each $(x, \alpha) \in X_{\mathcal{J}}^{0} \times \mathcal{A}$, the map

$$
\omega_{\alpha}(\cdot)+\varepsilon_{\alpha} J_{\alpha}(x, \cdot): T(x) \cap X_{\mathcal{J}}^{0} \rightarrow H \cup\{+\infty\}
$$

is $\left(T(x) \cap X_{\mathcal{J}}^{0}, X\right)$-lsc.

(ii) If $\mathcal{J}=\mathcal{P}$, then a special case of condition (C9) is a condition (C9") defined by:

$\left(\mathrm{C9}^{\prime \prime}\right)$ For each $(x, \alpha) \in X \times \mathcal{A}$, the map

$$
\omega_{\alpha}(\cdot)+\varepsilon_{\alpha} p_{\alpha}(x, \cdot): T(x) \rightarrow H \cup\{+\infty\}
$$

is $(T(x), X)$-lsc.

The proof will be broken into seven steps.

Step 1. Let $\Omega=\left\{\omega_{\alpha}: X \rightarrow L, \alpha \in \mathcal{A}\right\}$ where

$$
\forall_{\alpha \in \mathcal{A}} \forall_{x \in X}\left\{\omega_{\alpha}(x)=J_{\alpha}(x, T(x))\right\}
$$

The following hold:

$$
\forall_{\alpha \in \mathcal{A}} \forall_{x \in X}\left\{\left\{y \in T(x): \omega_{\alpha}(y) \preceq_{H} \lambda_{\alpha} J_{\alpha}(x, y)\right\}=T(x)\right\} ;
$$




$$
\begin{aligned}
& \forall_{\alpha \in \mathcal{A}} \forall_{x \in X}\left\{U_{\alpha}(x)=\left\{y \in T(x): \gamma_{\alpha} J_{\alpha}(x, y) \preceq_{H} \omega_{\alpha}(x)\right\} \neq \varnothing\right\} ; \\
& \forall_{\alpha \in \mathcal{A}} \forall_{x \in X}\left\{U_{\alpha}(x) \subset V_{\alpha}(x)=\left\{y \in T(x): \omega_{\alpha}(y)+\left(\gamma_{\alpha}-\lambda_{\alpha}\right) J_{\alpha}(x, y) \preceq_{H} \omega_{\alpha}(x)\right\}\right\} ;
\end{aligned}
$$

and

$$
\begin{aligned}
& \forall_{\alpha \in \mathcal{A}} \forall_{x \in X} \forall_{y \in T(x)}\left\{0 \preceq_{H} \omega_{\alpha}(x)-\omega_{\alpha}(y) \preceq_{H} \omega_{\alpha}(x)+\omega_{\alpha}(y)\right. \\
& \left.\quad \preceq_{H}\left(1+\lambda_{\alpha}\right) J_{\alpha}(x, y)\right\} .
\end{aligned}
$$

Indeed, by (A4), (A5) and (iii) and (iv) of Definition 2.6, we obtain

$$
\forall_{\alpha \in \mathcal{A}} \forall_{x, y \in X}\left\{\sup _{u \in T(x)} J_{\alpha}(u, T(y)) \preceq_{H} H_{\alpha}^{\mathcal{J}}(T(x), T(y)) \preceq_{H} \lambda_{\alpha} J_{\alpha}(x, y)\right\} .
$$

Hence, in particular, for $u=y$, we get

$$
\forall_{\alpha \in \mathcal{A}} \forall_{x \in X} \forall_{y \in T(x)}\left\{\omega_{\alpha}(y) \preceq_{H} \lambda_{\alpha} J_{\alpha}(x, y)\right\}
$$

This implies (4.1).

Note that

$$
\forall_{\alpha \in \mathcal{A}} \forall_{x \in X}\left\{J_{\alpha}(x, T(x))=\inf _{y \in T(x)} J_{\alpha}(x, y)\right\} .
$$

This, by (A6) (recall that $\left.\forall_{\alpha \in \mathcal{A}}\left\{\gamma_{\alpha} \in(0,1)\right\}\right)$, implies (4.2).

By (4.1) and (4.2), we have

$$
\begin{aligned}
& \forall_{\alpha \in \mathcal{A}} \forall_{x \in X}\left\{U_{\alpha}(x) \subset V_{\alpha}(x)=\left\{y \in T(x):\left(\gamma_{\alpha}-\lambda_{\alpha}\right) J_{\alpha}(x, y)\right.\right. \\
& \left.\left.\quad{ }_{H} J_{\alpha}(x, T(x))-J_{\alpha}(y, T(y))\right\}\right\},
\end{aligned}
$$

i.e., (4.3) holds.

By (4.3) and $(\mathcal{J} 1)$,

$$
\forall_{\alpha \in \mathcal{A}} \forall_{x \in X} \forall_{y \in T(x)}\left\{0 \preceq_{H} \omega_{\alpha}(x)-\omega_{\alpha}(y)\right\}
$$

and, by (4.5) and (4.1), we have

$$
\begin{gathered}
\forall_{\alpha \in \mathcal{A}} \forall_{x \in X} \forall_{y \in T(x)}\left\{\omega_{\alpha}(x)-\omega_{\alpha}(y) \preceq_{H} \omega_{\alpha}(x)+\omega_{\alpha}(y)\right. \\
\left.\quad=J_{\alpha}(x, T(x))+J_{\alpha}(y, T(y)) \preceq_{H}\left(1+\lambda_{\alpha}\right) J_{\alpha}(x, y)\right\} .
\end{gathered}
$$

Consequently, (4.4) holds.

Step 2. The family $\Omega$ defined in Step 1 satisfies (C4). Indeed, by (4.1),

$$
\forall_{x \in X}\left\{\left\{y \in T(x): \forall_{\alpha \in \mathcal{A}}\left\{\omega_{\alpha}(y) \preceq_{H} \lambda_{\alpha} J_{\alpha}(x, y)\right\}\right\}=T(x)\right\} .
$$

Also, by Definition 2.5, $\mathcal{J}=\left\{J_{\alpha}: X^{2} \rightarrow L, \alpha \in \mathcal{A}\right\},(\mathcal{J} 1)$ holds and, by (A4), $\forall_{x \in X}\{\varnothing \neq T(x)\}$. Hence, we conclude that $\forall_{x \in X}\left\{\varnothing \neq T(x) \subset D_{\Omega}\right\}$. 
Step 3. Assumptions (C8) and (C9) hold where $\forall_{\alpha \in \mathcal{A}}\left\{\varepsilon_{\alpha}=\gamma_{\alpha}-\lambda_{\alpha}\right\}$ and $\Omega$ is defined in Step 1.

By (4.2) and (4.3), in particular,

$$
\forall_{x \in X_{\mathcal{J}}^{0}} \forall_{\alpha \in \mathcal{A}}\left\{\varnothing \neq\left\{y \in T(x): \omega_{\alpha}(y)+\left(\gamma_{\alpha}-\lambda_{\alpha}\right) J_{\alpha}(x, y) \preceq_{H} \omega_{\alpha}(x)\right\}\right\}
$$

and, by (A7) and (A8), the following property concerning intersection of these sets holds: for each $x \in X_{\mathcal{J}}^{0}$,

$$
\left\{y \in T(x) \cap X_{\mathcal{J}}^{0}: \forall_{\alpha \in \mathcal{A}}\left\{\omega_{\alpha}(y)+\left(\gamma_{\alpha}-\lambda_{\alpha}\right) J_{\alpha}(x, y) \preceq_{H} \omega_{\alpha}(x)\right\}\right\}=Q_{\mathcal{J}, \Omega ; T}(x)
$$

is a nonempty closed subset in $X$.

Step 4. The assertions of Theorem 3.1 hold.

This follows from Assumptions (A1)-(A9), Steps 1-3, definition of $X_{\mathcal{J}}^{0}$ and Theorem 4.1. Step 5. Assumption (A10) implies (A7).

Indeed, denote

$$
\forall_{x \in X}\left\{U_{\mathcal{J}}(x)=\bigcap_{\alpha \in \mathcal{A}} U_{\alpha}(x)\right\}
$$

and

$$
\forall_{x \in X}\left\{V_{\mathcal{J}}(x)=\bigcap_{\alpha \in \mathcal{A}} V_{\alpha}(x)\right\} .
$$

By (4.2) and (4.3),

$$
\forall_{x \in X_{\mathcal{J}}^{0}}\left\{U_{\mathcal{J}}(x) \cap X_{\mathcal{J}}^{0} \subset V_{\mathcal{J}}(x) \cap X_{\mathcal{J}}^{0} \subset Q_{\mathcal{J} ; T}(x)\right\}
$$

Hence, we conclude that for each $x \in X_{\mathcal{J}}^{0}$, the set $Q_{\mathcal{J} ; T}(x)$ is nonempty whenever $\forall_{x \in X_{\mathcal{J}}^{0}}\left\{U_{\mathcal{J}}(x) \cap X_{\mathcal{J}}^{0} \neq \varnothing\right\}$

Step 6. Assumption (A11) implies (A8).

This follows from Remark 4.1(i)

Step 7. Assumption (A12) implies (A8).

Let $x_{0}$ be arbitrary and fixed, and let a sequence $\left(x_{m}: m \in \mathbb{N}\right)$ in $X$ be convergent to $x_{0}$, i.e., let $\forall_{\alpha \in \mathcal{A}}\left\{\lim _{m \rightarrow \infty} p_{\alpha}\left(x_{0}, x_{m}\right)=0\right\}$ (see Definition 2.2 and Theorem 2.1). If $m \in \mathbb{N}, v \in T\left(x_{m}\right)$ and $\alpha \in \mathcal{A}$ are arbitrary and fixed, then by $(\mathcal{J} 1)$,

$$
\omega_{\alpha}\left(x_{0}\right)=J_{\alpha}\left(x_{0}, T\left(x_{0}\right)\right) \preceq_{H} J_{\alpha}\left(x_{0}, x_{m}\right)+J_{\alpha}\left(x_{m}, v\right)+J_{\alpha}\left(v, T\left(x_{0}\right)\right) .
$$

Since $v \in T\left(x_{m}\right)$ and $T$ satisfy (A5), this implies

$$
\begin{aligned}
\omega_{\alpha}\left(x_{0}\right) & \preceq_{H} J_{\alpha}\left(x_{0}, x_{m}\right)+J_{\alpha}\left(x_{m}, T\left(x_{m}\right)\right)+\sup _{v \in T\left(x_{m}\right)} J_{\alpha}\left(v, T\left(x_{0}\right)\right) \\
& \preceq_{H} J_{\alpha}\left(x_{0}, x_{m}\right)+J_{\alpha}\left(x_{m}, T\left(x_{m}\right)\right)+H_{\alpha}^{\mathcal{J}}\left(T\left(x_{m}\right), T\left(x_{0}\right)\right) \\
& \preceq_{H} J_{\alpha}\left(x_{0}, x_{m}\right)+\omega_{\alpha}\left(x_{m}\right)+\lambda_{\alpha} J_{\alpha}\left(x_{m}, x_{0}\right),
\end{aligned}
$$


that is,

$$
0 \preceq_{H} J_{\alpha}\left(x_{0}, x_{m}\right)+\omega_{\alpha}\left(x_{m}\right)+\lambda_{\alpha} J_{\alpha}\left(x_{m}, x_{0}\right)-\omega_{\alpha}\left(x_{0}\right)
$$

Hence, by (A4), since $H$ is closed, using the fact that $\mathcal{J}$ is continuous and taking the limit as $m \rightarrow \infty$, we get

$$
0 \preceq_{H} \liminf _{m \rightarrow \infty} \omega_{\alpha}\left(x_{m}\right)-\omega_{\alpha}\left(x_{0}\right) .
$$

Therefore, for each $\alpha \in \mathcal{A}$,

$$
\omega_{\alpha}\left(x_{0}\right) \preceq_{H} \liminf _{m \rightarrow \infty} \omega_{\alpha}\left(x_{m}\right)
$$

i.e., $\omega_{\alpha}$ is lsc in $X$. Moreover, if $m \in \mathbb{N}, x \in X$ and $\alpha \in \mathcal{A}$ are arbitrary and fixed, then by $(\mathcal{J} 1)$

$$
J_{\alpha}\left(x, x_{0}\right) \preceq_{H} J_{\alpha}\left(x, x_{m}\right)+J_{\alpha}\left(x_{m}, x_{0}\right),
$$

that is,

$$
0 \preceq_{H} J_{\alpha}\left(x, x_{m}\right)+J_{\alpha}\left(x_{m}, x_{0}\right)-J_{\alpha}\left(x, x_{0}\right) .
$$

Since $H$ is closed and $\mathcal{J}$ is continuous, this implies

$$
0 \preceq_{H} \liminf _{m \rightarrow \infty} J_{\alpha}\left(x, x_{m}\right)-J_{\alpha}\left(x, x_{0}\right),
$$

that is, for each $(x, \alpha) \in X \times \mathcal{A}$, the map $J_{\alpha}(x, \cdot)$ is lsc in $X$. Hence, in particular, we conclude that for each $(x, \alpha) \in X_{\mathcal{J}}^{0} \times \mathcal{A}$, the map

$$
\omega_{\alpha}(\cdot)+\left(\gamma_{\alpha}-\lambda_{\alpha}\right) J_{\alpha}(x, \cdot): T(x) \cap X_{\mathcal{J}}^{0} \rightarrow H \cup\{+\infty\}
$$

is $\left(T(x) \cap X_{\mathcal{J}}^{0}, X\right)$-lsc, that is, $\left(\mathrm{C}^{\prime}\right)$ holds.

\section{Remarks, examples and comparisons}

Remark 5.1 Examples 5.1 and 5.2 illustrate a fixed point version and an endpoint version of Theorem 3.1, respectively, in cone metric spaces with $\mathcal{J}$-family where $\mathcal{J}=\{J\}$ and $J \neq p$.

\section{Example 5.1 If}

$$
X=\{\mathbf{N}=(n, n): n \in\{1,2,3,4,5,6\}\}=\{\mathbf{1}, \mathbf{2}, \mathbf{3}, \mathbf{4}, \mathbf{5}, \mathbf{6}\},
$$

$L=\mathbb{R}^{2}, H=\{(x, y) \in L: x, y \geq 0\} \subset \mathbb{R}^{2}$ and, for each $\beta>0, p: X^{2} \rightarrow L$ is defined by the formula

$$
p(\mathbf{N}, \mathbf{M})=(|n-m|, \beta|n-m|), \quad \mathbf{N}=(n, n), \mathbf{M}=(m, m) \in X,
$$

then $(X, \mathcal{P}), \mathcal{P}=\{p\}$ is a cone metric space; let in the sequel $\beta=2$. 
Let $T: X \rightarrow \mathrm{Cl}(X)$ be of the form:

$$
T(\mathbf{N})= \begin{cases}\{\mathbf{1}, \mathbf{2}\} & \text { if } \mathbf{N} \in X \backslash\{\mathbf{6}\} \\ \{\mathbf{4}, \mathbf{5}\} & \text { if } \mathbf{N}=\mathbf{6}\end{cases}
$$

Let $W=\{\mathbf{1}, \mathbf{2}, \mathbf{4}, \mathbf{5}\}$, and let $J: X^{2} \rightarrow L$ be of the form:

$$
J(\mathbf{N}, \mathbf{M})= \begin{cases}p(\mathbf{N}, \mathbf{M}) & \text { if }\{\mathbf{N}, \mathbf{M}\} \cap W=\{\mathbf{N}, \mathbf{M}\}, \\ (8,8)=\mathbf{8} & \text { if }\{\mathbf{N}, \mathbf{M}\} \cap W \neq\{\mathbf{N}, \mathbf{M}\},\end{cases}
$$

$\mathbf{N}, \mathbf{M} \in X$. Clearly, $\mathcal{J}=\{J\}$ is a $\mathcal{J}$-family on $X$ (see [32, Ex. 5.1]).

We observe that $X_{J}^{0}=\{\mathbf{1}, \mathbf{2}, \mathbf{4}, \mathbf{5}\} \neq \emptyset$.

We show that $(X, T)$ is $\mathcal{J}$-admissible and $\mathcal{H}_{3 / 4}^{J}$-contractive on $X$ where

$$
\forall_{A, B \in \mathrm{Cl}(X)}\left\{H^{J}(A, B)=\max \left\{\sup _{\mathbf{N} \in A} J(\mathbf{N}, B), \sup _{\mathbf{M} \in B} J(\mathbf{M}, A)\right\}\right\} .
$$

Indeed, let $\lambda=3 / 4$, and let $\mathbf{N}, \mathbf{M} \in X$ be arbitrary and fixed.

We consider three cases:

Case 1. If $\mathbf{N}, \mathbf{M} \in X \backslash\{\mathbf{6}\}$, then by definition of $T$, we have that $T(\mathbf{N})=T(\mathbf{M})=\{\mathbf{1}, \mathbf{2}\}$ and

$$
H^{J}(T(\mathbf{N}), T(\mathbf{M}))=(0,0)=\mathbf{0} \preceq_{H}(3 / 4) J(\mathbf{N}, \mathbf{M})=\lambda J(\mathbf{N}, \mathbf{M}) .
$$

Case 2. If $\mathbf{N} \in X \backslash\{\mathbf{6}\}$ and $\mathbf{M}=\mathbf{6}$, then by definition of $T, T(\mathbf{N})=\{\mathbf{1}, \mathbf{2}\}$ and $T(\mathbf{M})=\{\mathbf{4}, \mathbf{5}\}$. Hence, by definition of $J$, we calculate:

(i) $J(\mathbf{1}, T(\mathbf{M}))=p(\mathbf{1},\{\mathbf{4}, \mathbf{5}\})=(3,6), J(\mathbf{2}, T(\mathbf{M}))=p(\mathbf{2},\{\mathbf{4}, \mathbf{5}\})=(2,4)$ and

$$
\sup \{J(\mathbf{U}, T(\mathbf{M})): \mathbf{U} \in T(\mathbf{N})\}=(3,6)
$$

(ii) $J(\mathbf{4}, T(\mathbf{N}))=p(\mathbf{4},\{\mathbf{1}, \mathbf{2}\})=(2,4), J(\mathbf{5}, T(\mathbf{N}))=p(\mathbf{5},\{\mathbf{1}, \mathbf{2}\})=(3,6)$ and

$$
\sup \{J(\mathbf{V}, T(\mathbf{N})): \mathbf{V} \in T(\mathbf{M})\}=(3,6)
$$

(iii) By (i) and (ii),

$$
\begin{aligned}
H^{J}(T(\mathbf{N}), T(\mathbf{M}))= & \max \{\sup \{J(\mathbf{U}, T(\mathbf{M})): \mathbf{U} \in T(\mathbf{N})\}, \\
& \sup \{J(\mathbf{V}, T(\mathbf{N})): \mathbf{V} \in T(\mathbf{M})\}\}=(3,6) .
\end{aligned}
$$

Consequently,

$$
H^{J}(T(\mathbf{N}), T(\mathbf{M}))=(3,6) \preceq_{H} \mathbf{6}=(3 / 4) \cdot \mathbf{8}=\lambda J(\mathbf{N}, \mathbf{M})
$$

for $\mathbf{N} \in X \backslash\{\mathbf{6}\}$ and $\mathbf{M}=\mathbf{6}$.

Case 3. If $\mathbf{N}=\mathbf{6}$ and $\mathbf{M} \in X \backslash\{\mathbf{6}\}$, then by analogous considerations as in Case 2, we get

$$
H^{J}(T(\mathbf{N}), T(\mathbf{M}))=(3,6) \preceq_{H} \mathbf{6}=(3 / 4) \cdot \mathbf{8}=\lambda J(\mathbf{N}, \mathbf{M}) .
$$


Thus, $T$ is $\mathcal{J}$-admissible and $\mathcal{H}_{3 / 4}^{J}$-contractive on $X$.

Let now $\gamma=7 / 8$. Then for each $\mathbf{N} \in X_{J}^{0}=\{\mathbf{1}, \mathbf{2}, \mathbf{4}, \mathbf{5}\}$, we have $T(\mathbf{N})=\{\mathbf{1}, \mathbf{2}\}$ and using the fact that $T\left(X_{J}^{0}\right) \subset X_{J}^{0}$, we obtain

$$
\begin{aligned}
Q_{J ; T}(\mathbf{N}) & =\left\{\mathbf{M} \in\{\mathbf{1}, \mathbf{2}\}: J(\mathbf{M}, T(\mathbf{M}))+(1 / 8) J(\mathbf{N}, \mathbf{M}) \preceq_{H} J(\mathbf{N}, T(\mathbf{N}))\right\} \\
& =\left\{\mathbf{M} \in\{\mathbf{1}, \mathbf{2}\}:(1 / 8) p(\mathbf{N}, \mathbf{M}) \preceq_{H} p(\mathbf{N},\{\mathbf{1}, \mathbf{2}\})\right\} .
\end{aligned}
$$

This implies that

$$
\begin{aligned}
& Q_{J ; T}(\mathbf{1})=\left\{\mathbf{M} \in\{\mathbf{1}, \mathbf{2}\}:(1 / 8) p(\mathbf{1}, \mathbf{M}) \preceq_{H} \mathbf{0}\right\}=\{\mathbf{1}\}, \\
& Q_{J ; T}(\mathbf{2})=\left\{\mathbf{M} \in\{\mathbf{1}, \mathbf{2}\}:(1 / 8) p(\mathbf{2}, \mathbf{M}) \preceq_{H} \mathbf{0}\right\}=\{\mathbf{2}\}
\end{aligned}
$$

and

$$
Q_{J ; T}(\mathbf{N})=\left\{\mathbf{M} \in\{\mathbf{1}, \mathbf{2}\}:(1 / 8) p(\mathbf{N}, \mathbf{M}) \preceq_{H}(n-2,2 n-4)\right\}=\{\mathbf{1}, \mathbf{2}\}
$$

for $\mathbf{N}=(n, n) \in\{\mathbf{4}, \mathbf{5}\}$.

Assumptions (A1)-(A8) of Theorem 3.1 hold, Fix $(T)=\{(1,1),(2,2)\}$ and $J((1,1),(1,1))=$ $J((2,2),(2,2))=0$.

Example 5.2 Let $X, W, J, \lambda$ and $\gamma$ be such as in Example 5.1, and let $T: X \rightarrow \mathrm{Cl}(X)$ be of the form:

$$
T(\mathbf{N})= \begin{cases}\{\mathbf{1}\} & \text { if } \mathbf{N} \in\{\mathbf{1}, \mathbf{3}, \mathbf{5}\} \\ \{\mathbf{2}\} & \text { if } \mathbf{N} \in\{\mathbf{2}, \mathbf{4}\}, \\ \{\mathbf{4}, \mathbf{5}\} & \text { if } \mathbf{N}=\{\mathbf{6}\} .\end{cases}
$$

Then $X_{J}^{0}=\{\mathbf{1}, \mathbf{2}, \mathbf{4}, \mathbf{5}\}$ and

$$
Q_{J ; T}(\mathbf{N})=\left\{\mathbf{M} \in T(\mathbf{N}): J(\mathbf{M}, T(\mathbf{M}))+(1 / 8) J(\mathbf{N}, \mathbf{M}) \preceq_{H} J(\mathbf{N}, T(\mathbf{N}))\right\}
$$

for $\mathbf{N} \in X_{J}^{0}$ since $T\left(X_{J}^{0}\right) \subset X_{J}^{0}$. Hence:

$$
\begin{aligned}
& Q_{J ; T}(\mathbf{1})=\left\{\mathbf{M} \in\{\mathbf{1}\}: J(\mathbf{1}, \mathbf{1})+(1 / 8) J(\mathbf{1}, \mathbf{1}) \preceq_{H} J(\mathbf{1}, \mathbf{1})\right\}=\{\mathbf{1}\} ; \\
& Q_{J ; T}(\mathbf{2})=\left\{\mathbf{M} \in\{\mathbf{2}\}: J(\mathbf{2}, \mathbf{2})+(1 / 8) J(\mathbf{2}, \mathbf{2}) \preceq_{H} J(\mathbf{2}, \mathbf{2})\right\}=\{\mathbf{2}\} ; \\
& Q_{J ; T}(\mathbf{4})=\left\{\mathbf{M} \in\{\mathbf{2}\}: J(\mathbf{2}, \mathbf{2})+(1 / 8) J(\mathbf{4}, \mathbf{2}) \preceq_{H} J(\mathbf{4}, \mathbf{2})\right\}=\{\mathbf{2}\} ;
\end{aligned}
$$

and

$$
Q_{J ; T}(\mathbf{5})=\left\{\mathbf{M} \in\{\mathbf{1}\}: J(\mathbf{1}, \mathbf{1})+(1 / 8) J(\mathbf{5}, \mathbf{1}) \preceq_{H} J(\mathbf{5}, \mathbf{1})\right\}=\{\mathbf{1}\} .
$$

Assumptions (A1)-(A9) of Theorem 3.1 hold, $\operatorname{End}(T)=\{(1,1),(2,2)\}$ and $J((1,1),(1,1))=$ $J((2,2),(2,2))=0$. 
Remark 5.2 In Example 5.3, we show that in our concept of $\mathcal{H}_{\Lambda}^{\mathcal{J}}$-contractive set-valued dynamic systems, the existence of $\mathcal{J}$-family such that $\mathcal{J} \neq \mathcal{D}$ is essential; from Example 5.3, it follows that for maps defined in Examples 5.1 and 5.2, we cannot use Theorem 3.1 when $\mathcal{J}=\{p\}$.

Example 5.3 (a) Let $X$ and $T$ be such as in Example 5.1. We observe that for each $\lambda \in(0,1)$, $T$ is not $\mathcal{H}_{\lambda}^{p}$-contractive on $X$.

Otherwise, $J=p, X_{p}^{0}=X$ and

$$
\exists_{\lambda \in(0,1)} \forall_{\mathbf{N}, \mathbf{M} \in X}\left\{H^{p}(T(\mathbf{N}), T(\mathbf{M})) \leq \lambda p(\mathbf{N}, \mathbf{M})\right\} .
$$

However, for $\mathbf{N}_{0}=\mathbf{3}$ and $\mathbf{M}_{0}=\mathbf{6}$ from $X$, we obtain:

(i) $T\left(\mathbf{N}_{0}\right)=\{\mathbf{1}, \mathbf{2}\}$ and $T\left(\mathbf{M}_{0}\right)=\{\mathbf{4}, \mathbf{5}\}$;

(ii) $p\left(\mathbf{1}, T\left(\mathbf{M}_{0}\right)\right)=p(\mathbf{1},\{\mathbf{4}, \mathbf{5}\})=(3,6), p\left(\mathbf{2}, T\left(\mathbf{M}_{0}\right)\right)=p(\mathbf{2},\{\mathbf{4}, \mathbf{5}\})=(2,4)$ and

$$
\sup \left\{p\left(\mathbf{U}, T\left(\mathbf{M}_{0}\right)\right): \mathbf{U} \in T\left(\mathbf{N}_{0}\right)\right\}=(3,6)
$$

(iii) $p\left(\mathbf{4}, T\left(\mathbf{N}_{0}\right)\right)=p(\mathbf{4},\{\mathbf{1}, \mathbf{2}\})=(2,4), p\left(\mathbf{5}, T\left(\mathbf{N}_{0}\right)\right)=p(\mathbf{5},\{\mathbf{1}, \mathbf{2}\})=(3,6)$ and

$$
\sup \left\{p\left(\mathbf{V}, T\left(\mathbf{N}_{0}\right)\right): \mathbf{V} \in T\left(\mathbf{M}_{0}\right)\right\}=(3,6)
$$

(iv) By (i)-(iii),

$$
\begin{aligned}
H^{p}\left(T\left(\mathbf{N}_{0}\right), T\left(\mathbf{M}_{0}\right)\right)= & \max \left\{\sup \left\{p\left(\mathbf{U}, T\left(\mathbf{M}_{0}\right)\right): \mathbf{U} \in T\left(\mathbf{N}_{0}\right)\right\},\right. \\
& \left.\sup \left\{p\left(\mathbf{V}, T\left(\mathbf{N}_{0}\right)\right): \mathbf{V} \in T\left(\mathbf{M}_{0}\right)\right\}\right\}=(3,6) .
\end{aligned}
$$

Consequently, for each $\lambda \in(0,1)$,

$$
\begin{aligned}
(3,6) & =H^{p}\left(T\left(\mathbf{N}_{0}\right), T\left(\mathbf{M}_{0}\right)\right) \preceq_{H} \lambda p\left(\mathbf{N}_{0}, \mathbf{M}_{0}\right) \prec_{H} p\left(\mathbf{N}_{0}, \mathbf{M}_{0}\right) \\
& =p(\mathbf{3}, \mathbf{6})=(3,6) .
\end{aligned}
$$

It is absurd.

(b) Let $X$ and $T$ be such as in Example 5.2. By similar argumentation as in (a), we observe that for each $\lambda \in(0,1), T$ is not $\mathcal{H}_{\lambda}^{p}$-contractive on $X$.

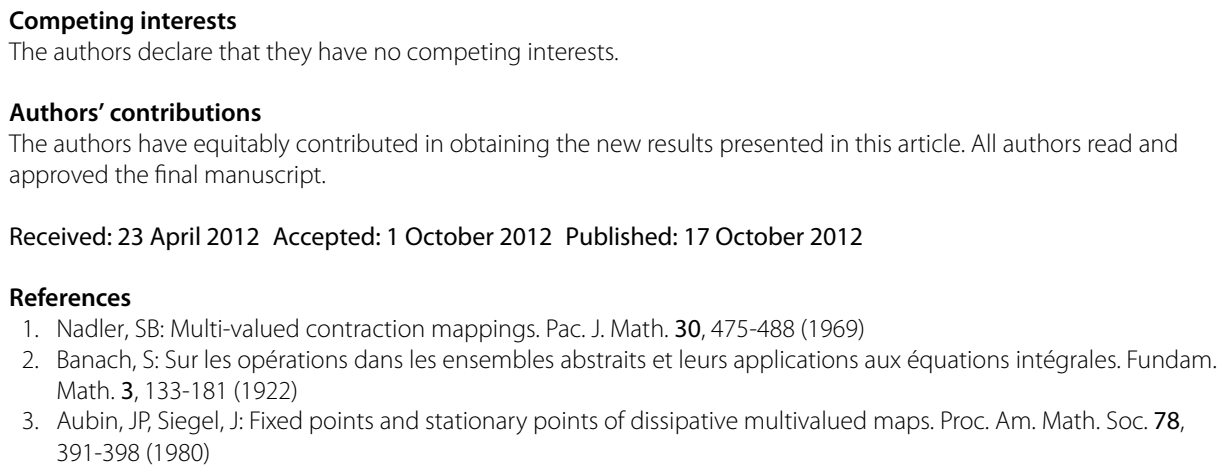

2. Banach, S: Sur les opérations dans les ensembles abstraits et leurs applications aux équations intégrales. Fundam. Math. 3, 133-181 (1922)

3. Aubin, JP, Siegel, J: Fixed points and stationary points of dissipative multivalued maps. Proc. Am. Math. Soc. 78, 391-398 (1980) 
4. de Blasi, FS, Myjak, J, Reich, S, Zaslawski, AJ: Generic existence and approximation of fixed points for nonexpansive set-valued maps. Set-Valued Var. Anal. 17, 97-112 (2009)

5. Ćirić, L: Multi-valued nonlinear contraction mappings. Nonlinear Anal. 71, 2716-2723 (2009)

6. Eldred, A, Anuradha, J, Veeramani, P: On the equivalence of the Mizoguchi-Takahashi fixed point theorem to Nadler's theorem. Appl. Math. Lett. 22, 1539-1542 (2009)

7. Feng, Y, Liu, S: Fixed point theorems for multi-valued contractive mappings and multi-valued Caristi type mappings. J. Math. Anal. Appl. 317, 103-112 (2006)

8. Frigon, M: Fixed point results for multivalued maps in metric spaces with generalized inwardness conditions. Fixed Point Theory Appl. 2010, Article ID 183217 (2010)

9. Al-Homidan, S, Ansari, QH, Yao, J-C: Some generalizations of Ekeland-type variational principle with applications to equilibrium problems and fixed point theory. Nonlinear Anal. 69, 126-139 (2008)

10. Jachymski, J: Caristi's fixed point theorem and selections of set-valued contractions. J. Math. Anal. Appl. 227, 55-67 (1998)

11. Kaneko, H: Generalized contractive multi-valued mappings and their fixed points. Math. Jpn. 33, 57-64 (1988)

12. Klim, D, Wardowski, A: Fixed point theorems for set-valued contractions in complete metric spaces. J. Math. Anal. Appl. 334, 132-139 (2007)

13. Latif, A, Al-Mezel, SA: Fixed point results in quasimetric spaces. Fixed Point Theory Appl. 2011, Article ID 178306 (2011)

14. Mizoguchi, N, Takahashi, W: Fixed point theorems for multivalued mappings on complete metric spaces. J. Math. Anal. Appl. 141, 177-188 (1989)

15. Pathak, HK, Shahzad, N: Fixed point results for set-valued contractions by altering distances in complete metric spaces. Nonlinear Anal. 70, 2634-2641 (2009)

16. Quantina, K, Kamran, T: Nadler's type principle with hight order of convergence. Nonlinear Anal. 69, 4106-4120 (2008)

17. Reich, S: Fixed points of contractive functions. Boll. Unione Mat. Ital. 4, 26-42 (1972)

18. Reich, S: Some problems and results in fixed point theory. In: Topological Methods in Nonlinear Functional Analysis. Contemp. Math., vol. 21, pp. 179-187. Am. Math. Soc., Providence (1983)

19. Reich, S, Zaslavski, AJ: Convergence of iterates of nonexpansive set-valued mappings. In: Set-valued Mappings with Applications in Nonlinear Analysis. Ser. Math. Anal. Appl., vol. 4, pp. 411-420. Taylor and Francis, London (2002)

20. Reich, S, Zaslavski, AJ: Generic existence of fixed points for set-valued mappings. Set-Valued Anal. 10, $287-296$ (2002)

21. Sintunavarat, $W$, Kumam, $P$ : Weak condition for generalized multi-valued $(f, \alpha, \beta)$-weak contraction mappings. Appl. Math. Lett. 24, 460-465 (2011)

22. Sintunavarat, W, Kumam, P: Gregus-type common fixed point theorems for tangential multivalued mappings of integral type in metric spaces. Int. J. Math. Math. Sci. 2011, Article ID 923458 (2011)

23. Sintunavarat, W, Kumam, P: Gregus type fixed points for a tangential multi-valued mappings satisfying contractive conditions of integral type. J. Inequal. Appl. 2011, 3 (2011)

24. Sintunavarat, W, Kumam, P: Common fixed point theorems for hybrid generalized multi-valued contraction mappings. Appl. Math. Lett. 25, 52-57 (2012)

25. Sintunavarat, W, Kumam, P: Common fixed point theorem for cyclic generalized multi-valued contraction mappings. Appl. Math. Lett. 25, 1849-1855 (2012)

26. Suzuki, T: Mizoguchi-Takahashi's fixed point theorem is a real generalization of Nadler's. J. Math. Anal. Appl. 340, 752-755 (2008)

27. Suzuki, T, Takahashi, W: Fixed point theorems and characterizations of metric completeness. Topol. Methods Nonlinear Anal. 8, 371-382 (1997)

28. Takahashi, W: Existence theorems generalizing fixed point theorems for multivalued mappings. In: Baillon, JB, Théra, M (eds.) Fixed Point Theory and Applications (Marseille, 1989). Pitman Res. Notes Math. Ser., vol. 252, pp. 397-406. Longman Sci. Tech., Harlow (1991)

29. Zhong, C-H, Zhu, J, Zhao, P-H: An extension of multi-valued contraction mappings and fixed points. Proc. Am. Math. Soc. 128, 2439-2444 (1999)

30. Suzuki, T: Several fixed point theorems concerning $\tau$-distance. Fixed Point Theory Appl. 2004, 195-209 (2004)

31. Wardowski, D: On set-valued contractions of Nadler type in cone metric spaces. Appl. Math. Lett. 24, 275-278 (2011)

32. Włodarczyk, K, Plebaniak, R: Maximality principle and general results of Ekeland and Caristi types without lower semicontinuity assumptions in cone uniform spaces with generalized pseudodistances. Fixed Point Theory Appl. 2010, Article ID 175453 (2010)

33. Tataru, D: Viscosity solutions of Hamilton-Jacobi equations with unbounded nonlinear terms. J. Math. Anal. Appl. 163 345-392 (1992)

34. Kada, O, Suzuki, T, Takahashi, W: Nonconvex minimization theorems and fixed point theorems in complete metric spaces. Math. Jpn. 44, 381-391 (1996)

35. Suzuki, T: Generalized distance and existence theorems in complete metric spaces. J. Math. Anal. Appl. 253, 440-458 (2001)

36. Lin, L-J, Du, W-S: Ekeland's variational principle, minimax theorems and existence of nonconvex equilibria in complete metric spaces. J. Math. Anal. Appl. 323, 360-370 (2006)

37. Vályi, I: A general maximality principle and a fixed point theorem in uniform spaces. Period. Math. Hung. 16, 127-134 (1985)

38. Włodarczyk, K, Plebaniak, R, Doliński, M: Cone uniform, cone locally convex and cone metric spaces, endpoints, set-valued dynamic systems and quasi-asymptotic contractions. Nonlinear Anal. 7(1), 5022-5031 (2009)

39. Włodarczyk, K, Plebaniak, R: Periodic point, endpoint, and convergence theorems for dissipative set-valued dynamic systems with generalized pseudodistances in cone uniform and uniform spaces. Fixed Point Theory Appl. 2010, Article ID 864536 (2010)

40. Włodarczyk, K, Plebaniak, R, Obczyński, C: Convergence theorems, best approximation and best proximity for set-valued dynamic systems of relatively quasi-asymptotic contractions in cone uniform spaces. Nonlinear Anal. 72 794-805 (2010)

41. Włodarczyk, K, Plebaniak, R: A fixed point theorem of Subrahmanyam type in uniform spaces with generalized pseudodistances. Appl. Math. Lett. 24, 325-328 (2011) 
42. Włodarczyk, K, Plebaniak, R: Quasi-gauge spaces with generalized quasi-pseudodistances and periodic points of dissipative set-valued dynamic systems. Fixed Point Theory Appl. 2011, Article ID 712706 (2011)

43. Włodarczyk, K, Plebaniak, R: Kannan-type contractions and fixed points in uniform spaces. Fixed Point Theory Appl. 2011, 90 (2011). doi:10.1186/1687-1812-2011-90

44. Włodarczyk, K, Plebaniak, R: Contractivity of Leader type and fixed points in uniform spaces with generalized pseudodistances. J. Math. Anal. Appl. 387, 533-541 (2012)

45. Włodarczyk, K, Plebaniak, R: Generalized uniform spaces, uniformly locally contractive set-valued dynamic systems and fixed points. Fixed Point Theory Appl. 2012, 104 (2012). doi:10.1186/1687-1812-2012-104

46. Włodarczyk, K, Plebaniak, R: Leader type contractions, periodic and fixed points and new completivity in quasi-gauge spaces with generalized quasi-pseudodistances. Topol. Appl. 159, 3504-3512 (2012)

47. Yuan, GX-Z: KKM Theory and Applications in Nonlinear Analysis. Marcel Dekker, New York (1999)

doi:10.1186/1687-1812-2012-176

Cite this article as: Włodarczyk and Plebaniak: Fixed points and endpoints of contractive set-valued maps in cone uniform spaces with generalized pseudodistances. Fixed Point Theory and Applications 2012 2012:176.

\section{Submit your manuscript to a SpringerOpen ${ }^{\circ}$ journal and benefit from:}

- Convenient online submission

- Rigorous peer review

- Immediate publication on acceptance

- Open access: articles freely available online

- High visibility within the field

- Retaining the copyright to your article 